\title{
ON SPECTRA AND BROWN'S SPECTRAL MEASURES OF ELEMENTS IN FREE PRODUCTS OF MATRIX ALGEBRAS
}

\author{
JUNSHENG FANG, DON HADWIN and XIUJUAN MA
}

\begin{abstract}
We compute spectra and Brown measures of some non self-adjoint operators in $\left(M_{2}(\mathrm{C}), \frac{1}{2} \mathrm{Tr}\right) *$ $\left(M_{2}(\mathrm{C}), \frac{1}{2} \mathrm{Tr}\right)$, the reduced free product von Neumann algebra of $M_{2}(\mathrm{C})$ with $M_{2}(\mathrm{C})$. Examples include $A B$ and $A+B$, where $A$ and $B$ are matrices in $\left(M_{2}(\mathrm{C}), \frac{1}{2} \operatorname{Tr}\right) * 1$ and $1 *\left(M_{2}(\mathrm{C}), \frac{1}{2} \operatorname{Tr}\right)$, respectively. We prove that $A B$ is an R-diagonal operator (in the sense of Nica and Speicher [12]) if and only if $\operatorname{Tr}(A)=\operatorname{Tr}(B)=0$. We show that if $X=A B$ or $X=A+B$ and $A, B$ are not scalar matrices, then the Brown measure of $X$ is not concentrated on a single point. By a theorem of Haagerup and Schultz [9], we obtain that if $X=A B$ or $X=A+B$ and $X \neq \lambda 1$, then $X$ has a nontrivial hyperinvariant subspace affiliated with $\left(M_{2}(\mathrm{C}), \frac{1}{2} \operatorname{Tr}\right) *\left(M_{2}(\mathrm{C}), \frac{1}{2} \operatorname{Tr}\right)$.
\end{abstract}

\section{Introduction}

In 1983, L. G. Brown [2] introduced a spectral distribution measure for nonnormal elements in a finite von Neumann algebra with respect to a fixed normal faithful tracial state, which is called the Brown measure of the operator. Recently, U. Haagerup and H. Schultz [9] proved a remarkable result which states that if the support of Brown measure of an operator in a type $\mathrm{II}_{1}$ factor contains more than two points, then the operator has a non-trivial hyperinvariant subspace affiliated with the type $\mathrm{II}_{1}$ factor. In general cases, the computation of Brown measures of non-normal operators are nontrivial. The first essential result was given by Haagerup and F. Larsen. In [8], Haagerup and Larsen computed the spectrum and Brown measure of R-diagonal operators in a finite von Neumann algebra, in terms of the distribution of its radial part. Brown measures of some non-normal and non-R-diagonal operators, examples include $u_{n}+u_{\infty}$, where $u_{n}$ and $u_{\infty}$ are the generators of $Z_{n}$ and $Z$ respectively, in the free product $\mathrm{Z}_{n} * \mathrm{Z}$, and elements of the form $S_{\alpha}+i S_{\beta}$, where $S_{\alpha}$ and $S_{\beta}$ are free semicircular elements of variance $\alpha$ and $\beta$, are computed by P. Biane and F. Lehner in [1]. The purpose of this paper is to compute the spectra and Brown measures of some non hermitian operators in $\left(M_{2}(\mathrm{C}), \frac{1}{2} \mathrm{Tr}\right) *\left(M_{2}(\mathrm{C}), \frac{1}{2} \mathrm{Tr}\right)$, the reduced free product von Neumann algebra of $M_{2}(\mathrm{C})$ with $M_{2}(\mathrm{C})$ (cf [3]). Examples 
include $A B$ and $A+B$, where $A$ and $B$ are matrices in $\left(M_{2}(C), \frac{1}{2} \operatorname{Tr}\right) * 1$ and $1 *\left(M_{2}(\mathrm{C}), \frac{1}{2} \mathrm{Tr}\right)$, respectively. This paper is organized as follows.

In section 2 we recall some preliminary facts about Brown measures, Rdiagonal operators, Haagerup and Larsen's result on Brown measures of Rdiagonal operators and some notation used in this paper. In section 3, we provide some results on the spectra and spectral radius of operators in $M_{2}(\mathrm{C}) *$ $M_{2}(\mathrm{C})$, the universal free product $\mathrm{C}^{*}$-algebra of $M_{2}(\mathrm{C})$ with $M_{2}(\mathrm{C})$. Firstly we compute the spectral radius of $A B$ for two normal matrices $A \in M_{2}(\mathrm{C}) * 1$ and $B \in 1 * M_{2}(\mathrm{C})$ relative to $M_{2}(\mathrm{C}) * M_{2}(\mathrm{C})$. As a corollary, we also get the spectrum radius of $A B$ for normal matrices $A \in\left(M_{2}(\mathrm{C}), \frac{1}{2} \operatorname{Tr}\right) * 1$ and $B \in$ $1 *\left(M_{2}(\mathrm{C}), \frac{1}{2} \mathrm{Tr}\right)$, relative to the reduced free product von Neumann algebra of $M_{2}(\mathrm{C})$ with $M_{2}(\mathrm{C})$. Then we obtain the following result: Let $A, B$ be matrices in $M_{2}(\mathrm{C}) * 1$ and $1 * M_{2}(\mathrm{C})$, respectively, such that $\operatorname{Tr}(A)=\operatorname{Tr}(B)=0$. Then $\sigma(A B)$, the spectrum of $A B$, relative to $M_{2}(\mathrm{C}) * M_{2}(\mathrm{C})$, is the closure of the annulus centered at 0 with inner radius $\left\|A^{-1}\right\|^{-1}\left\|B^{-1}\right\|^{-1}$ and outer radius $\|A\|\|B\|$, where we use the convention $\infty^{-1}=0$ and if $A$ is not invertible then $\left\|A^{-1}\right\|:=\infty$.

In section 4 we prove that $A B$ is an R-diagonal operator if and only if $\operatorname{Tr}(A)=\operatorname{Tr}(B)=0$, where $A \in\left(M_{2}(C), \frac{1}{2} \operatorname{Tr}\right) * 1$ and $B \in 1 *\left(M_{2}(C), \frac{1}{2} \operatorname{Tr}\right)$. As a corollary, we explicitly compute the spectrum and Brown measure of $A B$ $(\operatorname{Tr}(A)=\operatorname{Tr}(B)=0)$ in terms of S-transform of $A^{*} A$ and $B^{*} B$.

In section 5, we develop algebraic techniques used in [4]. Let $X \in 1 *$ $\left(M_{2}(\mathrm{C}), \frac{1}{2} \operatorname{Tr}\right)$. With respect to the matrix units of $\left(M_{2}(\mathrm{C}), \frac{1}{2} \operatorname{Tr}\right) * 1, X=$ $\left(\begin{array}{ll}x_{1} & x_{2} \\ x_{3} & x_{4}\end{array}\right)$. By [4], $\left(M_{2}(\mathrm{C}), \frac{1}{2} \operatorname{Tr}\right) *\left(M_{2}(\mathrm{C}), \frac{1}{2} \operatorname{Tr}\right) \cong L\left(\mathrm{~F}_{3}\right) \otimes M_{2}(\mathrm{C})$. So $x_{1}, x_{2}, x_{3}, x_{4} \in L\left(\mathrm{~F}_{3}\right)$. In section 5 , we find $*$-free generators $h, u, v$ of $L\left(\mathrm{~F}_{3}\right)$ (different from the free generators given in [4]) so that we may explicitly write out $x_{1}, x_{2}, x_{3}, x_{4}$ in terms of $h, u, v$.

In section 6, we compute miscellaneous examples of Brown measures of operators $A+B$ and $A B$, where $A \in\left(M_{2}(\mathrm{C}), \frac{1}{2} \operatorname{Tr}\right) * 1$ and $B \in 1 *\left(M_{2}(\mathrm{C}), \frac{1}{2} \mathrm{Tr}\right)$. As a corollary, we show that $A+B$ is an R-diagonal operator if and only if $A+B=0$.

In section 7, we prove the following result: Let $A \in\left(M_{2}(\mathrm{C}), \frac{1}{2} \mathrm{Tr}\right) * 1$ and $B \in 1 *\left(M_{2}(\mathrm{C}), \frac{1}{2} \operatorname{Tr}\right)$. if $X=A+B$ or $X=A B$ and $A, B$ are not scalar matrices, then the Brown measure of $X$ is not concentrated on a single point. As a corollary of Theorem 7.1 of [9], we prove that if $X=A+B$ or $X=A B$ and $X \neq \lambda 1$, then $X$ has a nontrivial hyperinvariant subspace affiliated with $\left(M_{2}(\mathrm{C}), \frac{1}{2} \operatorname{Tr}\right) *\left(M_{2}(\mathrm{C}), \frac{1}{2} \mathrm{Tr}\right)$.

Many concrete examples of spectra and Brown measures are given in this paper. For some interesting applications, we refer to [5]. 


\section{Preliminaries}

\subsection{Fuglede-Kadison determinant and Brown's spectral measure}

Let $\mathcal{M}$ be a finite von Neumann algebra with a faithful normal tracial state $\tau$. The Fuglede-Kadison determinant [6], $\Delta: \mathcal{M} \rightarrow[0, \infty[$, is given by

$$
\Delta(T)=\exp \{\tau(\log |T|)\}, \quad T \in \mathscr{M},
$$

with $\exp \{-\infty\}:=0$. For an arbitrary element $T$ in $\mathscr{M}$ the function $\lambda \rightarrow$ $\log \Delta(T-\lambda 1)$ is subharmonic on $\mathrm{C}$, and its Laplacian

$$
d \mu_{T}(\lambda):=\frac{1}{2 \pi} \nabla^{2} \log \Delta(T-\lambda 1),
$$

in the distribution sense, defines a probability measure $\mu_{T}$ on $C$, called the Brown's measure [2] of $T$. From the definition, Brown measure $\mu_{T}$ only depends on the joint distribution of $T$ and $T^{*}$.

If $T$ is normal, $\mu_{T}$ is the trace $\tau$ composed with the spectral projections of $T$. If $\mathscr{M}=M_{n}(\mathrm{C})$ and $\tau=\frac{1}{n} \mathrm{Tr}$ is the normalized trace on $M_{n}(\mathrm{C})$, then $\mu_{T}$ is the normalized counting measure $\frac{1}{n}\left(\delta_{\lambda_{1}}+\delta_{\lambda_{2}} \cdots+\delta_{\lambda_{n}}\right)$, where $\lambda_{1}, \lambda_{2} \cdots, \lambda_{n}$ are the eigenvalues of $T$ repeated according to root multiplicity.

The Brown measure has the following properties (see [2], [10]): $\mu_{T}$ is the unique compactly supported measure on $C$ such that $\log \Delta(T-\lambda 1)=$ $\int_{\mathrm{C}} \log |z-\lambda| d \mu_{T}(z)$ for all $\lambda \in \mathrm{C}$. The support of $\mu_{T}$ is contained in $\sigma(T)$, the spectrum of $T$. $\mu_{S T}=\mu_{T S}$ for arbitrary $S, T$ in $\mathscr{M}$, and if $f(z)$ is analytic in a neighborhood of $\sigma(T), \mu_{f(T)}=\left(\mu_{T}\right)_{f}$, the push-forward measure of $\mu_{T}$ under the map $\lambda \rightarrow f(\lambda)$. If $E \in \mathscr{M}$ is a projection such that $E \in \operatorname{Lat} T$, then with respect to $E, I-E$ we can write

$$
T=\left(\begin{array}{cc}
A & B \\
0 & C
\end{array}\right)
$$

where $A=E T E$ and $C=(I-E) T(I-E)$ are elements of $\mathscr{M}_{1}=E \mathscr{M} E$ and $\mathscr{M}_{2}=(I-E) \mathscr{M}(I-E)$, respectively. Let $\mu_{A}$ and $\mu_{C}$ be the Brown measures of $A$ and $C$ computed relative to $\mathscr{M}_{1}$ and $\mathscr{M}_{2}$, respectively. Then $\mu_{T}=\alpha \mu_{A}+(1-\alpha) \mu_{C}$, where $\alpha=\tau(E)$.

For a generalization of Brown measures of sets of commuting operators in a type $\mathrm{II}_{1}$ factor, we refer to [15].

\subsection{R-diagonal operators}

In 1995, A. Nica and S. Speicher [12] introduced the class of R-diagonal operators in non-commutative probability spaces. Recall that an operator $T$ 
in a non-commutative probability space is an R-diagonal operator if the $\mathrm{R}$ transform $R_{\mu\left(T, T^{*}\right)}$ of the joint distribution $\mu\left(T, T^{*}\right)$ of $T, T^{*}$ is of the form

$$
R_{\mu\left(T, T^{*}\right)}\left(z_{1}, z_{2}\right)=\sum_{n=1}^{\infty} \alpha_{n}\left(z_{1} z_{2}\right)^{n}+\sum_{n=1}^{\infty} \alpha_{n}\left(z_{2} z_{1}\right)^{n}
$$

Nica and Speicher [12] proved that $T$ is an $R$-diagonal operator if and only if $T$ has same $*$-distribution as product $U H$, where $U$ and $H$ are $*$-free random variables in some tracial non commutative probability space, $U$ is a Haar unitary operator and $H$ is positive. If $T$ is an R-diagonal operator, then the *-distribution of $T$ is uniquely determined by the distribution of $T^{*} T=|T|^{2}$. If $T$ is an R-diagonal operator and $S$ is $*$-free with $T$, then both $S T$ and $T S$ are R-diagonal operators (see [12]). If $T$ is an R-diagonal operator and $n \in \mathrm{N}$, then $T^{n}$ is also an R-diagonal operator (see [8], [11]). For other important properties of R-diagonal operators, we refer to [8], [11], [12], [13].

\subsection{Brown measures of R-diagonal operators}

In [8], Haagerup and Larson explicitly computed the Brown measures of Rdiagonal operators in a finite von Neumann algebra.

THEOREM 2.1 (Theorem 4.4 of [8]). Let $U, H$ be $*$-free random variables in a noncommutative probability space $(\mathcal{M}, \tau)$, with $U$ a Haar unitary operator and $H$ a positive operator such that the distribution $\mu_{H}$ of $H$ is not a Dirac measure. Then the Brown measure $\mu_{U H}$ of $U H$ can be computed as the following.

(1) $\mu_{U H}$ is rotation invariant and its support is the annulus with inner radius $\left\|H^{-1}\right\|_{2}^{-1}$ and outer radius $\|H\|_{2}$.

(2) $\mu_{U H}(\{0\})=\mu_{H}(\{0\})$ and for $\left.\left.t \in\right] \mu_{H}(\{0\}), 1\right]$,

$$
\mu_{U H}\left(\mathrm{~B}\left(0,\left(S_{\mu_{H^{2}}}(t-1)\right)^{-1 / 2}\right)\right)=t,
$$

where $S_{\mu_{H^{2}}}$ is the S-transform of $H^{2}$ and $\mathrm{B}(0, r)$ is the open disc with center 0 and radius $r$;

(3) $\mu_{U H}$ is the only rotation invariant symmetric probability measure satisfying (2).

Furthermore, if $H$ is invertible, then $\sigma(U H)=\operatorname{supp} \mu_{U H}$; if $H$ is not invertible, then $\sigma(U H)=\overline{\mathrm{B}\left(0,\|H\|_{2}\right)}$. 


\subsection{Some Notation}

The following notation will be used in the rest of the paper

- $(\mathscr{M}, \tau)=\left(M_{2}(\mathrm{C}), \frac{1}{2} \operatorname{Tr}\right) *\left(M_{2}(\mathrm{C}), \frac{1}{2} \operatorname{Tr}\right)$ denotes the reduced free product von Neumann algebra of $M_{2}(\mathrm{C})$ with $M_{2}(\mathrm{C})$ with the unique tracial state $\tau$;

- $M_{2}(\mathrm{C})_{(1)}:=\left(M_{2}(\mathrm{C}), \frac{1}{2} \mathrm{Tr}\right) * 1$ and $M_{2}(\mathrm{C})_{(2)}:=1 *\left(M_{2}(\mathrm{C}), \frac{1}{2} \mathrm{Tr}\right)$;

- $\left\{E_{i j}\right\}_{i, j=1,2},\left\{F_{i j}\right\}_{i, j=1,2}$ are matrix units of $M_{2}(\mathrm{C})_{(1)}$ and $M_{2}(\mathrm{C})_{(2)}$, respectively;

- $P=E_{11}$ and $Q=F_{11}$;

- $\mathcal{M} \cong \mathcal{N} \otimes M_{2}(\mathrm{C})_{(1)} \cong \mathcal{N} \otimes M_{2}(\mathrm{C})_{(2)}$. For $X \in \mathscr{M}, X=\left(\begin{array}{ll}x_{1} & x_{2} \\ x_{3} & x_{4}\end{array}\right)_{(1)}=$ $\left(\begin{array}{ll}x_{1}^{\prime} & x_{2}^{\prime} \\ x_{3}^{\prime} & x_{4}^{\prime}\end{array}\right)_{(2)}$ means the decomposition is with respect to above matrix units of $M_{2}(\mathrm{C})_{(1)}$ and $M_{2}(\mathrm{C})_{(2)}$, respectively.

$$
\begin{aligned}
\text { - } W_{0} & =\left(\begin{array}{ll}
1 & 0 \\
0 & 1
\end{array}\right)_{(1)}, W_{1}=\left(\begin{array}{rr}
1 & 0 \\
0 & -1
\end{array}\right)_{(1)}, W_{2}=\left(\begin{array}{rr}
0 & -1 \\
1 & 0
\end{array}\right)_{(1)}, \\
W_{3} & =\left(\begin{array}{ll}
0 & 1 \\
1 & 0
\end{array}\right)_{(1)} ; \\
\text { - } V_{0} & =\left(\begin{array}{ll}
1 & 0 \\
0 & 1
\end{array}\right)_{(2)}, V_{1}=\left(\begin{array}{rr}
1 & 0 \\
0 & -1
\end{array}\right)_{(2)}, V_{2}=\left(\begin{array}{rr}
0 & -1 \\
1 & 0
\end{array}\right)_{(2)}, \\
V_{3} & =\left(\begin{array}{ll}
0 & 1 \\
1 & 0
\end{array}\right)_{(2)} ;
\end{aligned}
$$

- $A, A_{1}, \ldots, A_{n}$ denote elements in $M_{2}(\mathrm{C})_{(1)}, B, B_{1}, \ldots, B_{n}$ denote elements in $M_{2}(\mathrm{C})_{(2)}, X, Y, Z$ denote general elements in $\mathscr{M}$;

- An element $X$ in $\mathscr{M}$ is called centered if $\tau(X)=0$.

We end this section with the following lemma. The proof is an easy exercise.

Lemma 2.2. $V_{1} M_{2}(\mathrm{C})_{(1)} V_{1}$ is free with $M_{2}(\mathrm{C})_{(1)}$.

\section{Spectra of elements in the universal free product of $M_{2}(C)$ and $M_{2}(\mathrm{C})$}

Let $\AA=M_{2}(\mathrm{C}) * M_{2}(\mathrm{C})$ denote the universal free product $\mathrm{C} *$-algebra of $M_{2}(\mathrm{C})$ with $M_{2}(\mathrm{C})$. Then there is a ${ }^{*}$ homomorphism $\pi$ from $\AA$ onto the reduced free product $\mathrm{C}^{*}$-algebra of $M_{2}(\mathrm{C})$ and $M_{2}(\mathrm{C})$, the $\mathrm{C}^{*}$-subalgebra generated by $M_{2}(\mathrm{C})_{(1)}$ and $M_{2}(\mathrm{C})_{(2)}$ in $\mathcal{M}$. Since $\sigma(\pi(a)) \subseteq \sigma(a)$ for $a \in \AA$, it is useful to obtain some information of spectrum of $A B$, where $A \in M_{2}(\mathrm{C}) * 1$ and $B \in 1 * M_{2}(\mathrm{C})$. 


\section{1. "Free product" of normal matrices}

Lemma 3.1. Let $A \in M_{2}(\mathrm{C}) * 1$ and $B \in 1 * M_{2}(\mathrm{C})$ be normal matrices. Then $r(A B)=\|A\| \cdot\|B\|$ relative to $\AA$.

Proof. $r(A B) \leq\|A B\| \leq\|A\| \cdot\|B\|$. We need only to prove $r(A B) \geq$ $\|A\| \cdot\|B\|$. Since $A$ is a normal matrix, there is a unitary matrix $U_{1} \in M_{2}(\mathrm{C}) * 1$ such that $U_{1} A U_{1}^{*}=\left(\begin{array}{cc}\alpha_{1} & 0 \\ 0 & \beta_{1}\end{array}\right)$ and $\left\|\alpha_{1}\right\|=\|A\|$. Similarly, there is a unitary matrix $U_{2} \in 1 * M_{2}(\mathrm{C})$ such that $U_{2} B U_{2}^{*}=\left(\begin{array}{cc}\alpha_{2} & 0 \\ 0 & \beta_{2}\end{array}\right)$ and $\left\|\alpha_{2}\right\|=\|B\|$. Let $\pi_{1}(X)=U_{1} X U_{1}^{*}$ and $\pi_{2}(Y)=U_{2} Y U_{2}^{*}$ be $*$-representations of $M_{1}(\mathrm{C}) * 1$ and $1 * M_{2}(\mathrm{C})$ to $M_{2}(\mathrm{C})$, respectively. Then there is a $*$-representation $\pi=$ $\pi_{1} * \pi_{2}$ from $\AA$ to $M_{2}(\mathrm{C})$ and $\pi(A B)=\left(\begin{array}{cc}\alpha_{1} \alpha_{2} & 0 \\ 0 & \beta_{1} \beta_{2}\end{array}\right)$. Therefore, $\alpha_{1} \alpha_{2} \in$ $\sigma(\pi(A B)) \subseteq \sigma(A B)$. So $r(A B) \geq\left|\alpha_{1} \alpha_{2}\right|=\|A\| \cdot\|B\|$.

Corollary 3.2. Let $A \in M_{2}(\mathrm{C})_{(1)}$ and $B \in M_{2}(\mathrm{C})_{(2)}$ be normal matrices. Then $r(A B)=\|A\| \cdot\|B\|$ relative to $\mathcal{M}$.

Proof. We may assume that $A$ and $B$ are diagonal matrices. Then we can treat $A B$ as an operator in the full free product $C^{*}\left(\mathrm{Z}_{2} * \mathrm{Z}_{2}\right)$. Same technique used in the previous lemma gives the corollary.

\section{2. "Free product" of non-normal matrices}

It is well-known that two matrices $X, Y$ in $M_{2}(\mathrm{C})$ are unitarily equivalent if and only if $\operatorname{Tr}(X)=\operatorname{Tr}(Y), \operatorname{Tr}\left(X^{2}\right)=\operatorname{Tr}\left(Y^{2}\right)$ and $\operatorname{Tr}\left(X^{*} X\right)=\operatorname{Tr}\left(Y^{*} Y\right)$. The proof of the following lemma now is an easy exercise.

Lemma 3.3. If $A \in M_{2}(\mathrm{C})$ and $\operatorname{Tr}(A)=0$, then $A$ is unitarily equivalent to a matrix of form $\left(\begin{array}{ll}0 & \alpha \\ \beta & 0\end{array}\right)$, where $\alpha, \beta$ are complex numbers.

REMARK 3.4. We have the following useful observations:

$$
\begin{aligned}
& \text { - }\left(\begin{array}{ll}
0 & 1 \\
1 & 0
\end{array}\right)\left(\begin{array}{cc}
0 & \alpha \\
\beta & 0
\end{array}\right)\left(\begin{array}{ll}
0 & 1 \\
1 & 0
\end{array}\right)=\left(\begin{array}{ll}
0 & \beta \\
\alpha & 0
\end{array}\right) . \\
& \text { - }\left(\begin{array}{cc}
1 & 0 \\
0 & e^{i\left(\theta_{1}-\theta_{2}\right) / 2}
\end{array}\right)\left(\begin{array}{cc}
0 & |\alpha| e^{i \theta_{1}} \\
|\beta| e^{i \theta_{2}} & 0
\end{array}\right)\left(\begin{array}{ll}
1 & 0 \\
0 & e^{-i\left(\theta_{1}-\theta_{2}\right) / 2}
\end{array}\right)= \\
& e^{i\left(\theta_{1}+\theta_{2}\right) / 2}\left(\begin{array}{cc}
0 & |\alpha| \\
|\beta| & 0
\end{array}\right) .
\end{aligned}
$$

Lemma 3.5. Let $A \in M_{2}(\mathrm{C}) * 1$ and $B \in 1 * M_{2}(\mathrm{C})$ be matrices such that $\operatorname{Tr}(A)=\operatorname{Tr}(B)=0$. Then $r(A B)=\|A\| \cdot\|B\|$ relative to $\AA$. 
Proof. We need only to prove $r(A B) \geq\|A\| \cdot\|B\|$. By Lemma 3.3 and Remark 3.4, there are unitary matrices $U, V$ in $M_{2}(\mathrm{C})$ such that $U A U^{*}=$ $\left(\begin{array}{cc}0 & \alpha_{1} \\ \beta_{1} & 0\end{array}\right)$ and $V B V^{*}=\left(\begin{array}{cc}0 & \alpha_{2} \\ \beta_{2} & 0\end{array}\right)$ and $\left|\alpha_{1}\right|=\|A\|,\left|\beta_{2}\right|=\|B\|$. Let $\pi_{1}(X)=U X U^{*}$ and $\pi_{2}(Y)=V Y V^{*}$ be $*$-representations of $M_{2}(\mathrm{C}) * 1$ and $1 *$ $M_{2}(\mathrm{C})$ to $M_{2}(\mathrm{C})$, respectively. Let $\pi=\pi_{1} * \pi_{2}$ be the induced $*$-representation of $\AA$ to $M_{2}(C)$. Then $\sigma(A B) \supseteq \sigma(\pi(A B))=\sigma\left(\pi_{1}(A) \pi_{2}(B)\right)=\left\{\alpha_{1} \beta_{2}, \alpha_{2} \beta_{1}\right\}$. Therefore, $r(A B) \geq\left|\alpha_{1} \beta_{2}\right|=\|A\| \cdot\|B\|$.

Theorem 3.6. Let $A \in M_{2}(\mathrm{C}) * 1$ and $B \in 1 * M_{2}(\mathrm{C})$ be matrices such that $\operatorname{Tr}(A)=\operatorname{Tr}(B)=0$. Then

$$
\sigma(A B)=\left[\left\|A^{-1}\right\|^{-1}\left\|B^{-1}\right\|^{-1},\|A\|\|B\|\right] \times_{p}[0,2 \pi],
$$

where $\times_{p}$ denotes the polar set product $\left\{r e^{i \theta}: r \in\left[\left\|A^{-1}\right\|^{-1}\left\|B^{-1}\right\|^{-1}\right.\right.$, $\|A\|\|B\|], \theta \in[0,2 \pi]\}$.

Proof. We will prove the theorem for two cases.

Case 1. Either $A$ or $B$ is not invertible. We may assume that $A$ is not invertible. $\operatorname{By} \operatorname{Tr}(A)=0$, Lemma 3.3 and Remark 3.4, $A$ is unitarily equivalent to $\left(\begin{array}{cc}0 & \alpha_{1} \\ 0 & 0\end{array}\right)$. Without loss of generality, we assume that $A=\left(\begin{array}{ll}0 & 1 \\ 0 & 0\end{array}\right) \in$ $M_{2}(\mathrm{C}) * 1$. By Lemma 3.3 and Remark 3.4, we may also assume that $B=$ $\left(\begin{array}{ll}0 & \alpha \\ \beta & 0\end{array}\right) \in 1 * M_{2}(\mathrm{C})$ and $\beta \geq \alpha \geq 0$. We need to prove that $\sigma(A B)$ is the closed disc of complex plane with center 0 and radius $\beta$. Since $A$ is unitarily equivalent to $e^{i \theta} A$ in $M_{2}(\mathrm{C}) * 1, \sigma(A B)$ is rotation invariant. For $\theta \in[0,2 \pi]$, let $U=\left(\begin{array}{cc}\cos \theta & \sin \theta \\ -\sin \theta & \cos \theta\end{array}\right)$. Let $\pi_{1}(X)=X$ and $\pi_{2}(Y)=U Y U^{*}$ be $*$-representations of $M_{2}(\mathrm{C}) * 1$ and $1 * M_{2}(\mathrm{C})$ to $M_{2}(\mathrm{C})$, respectively. Let $\pi=\pi_{1} * \pi_{2}$ be the induced $*$-representation of $\AA$ to $M_{2}(\mathrm{C})$. Then

$$
\pi(A B)=A U B U^{*}=\left(\begin{array}{cc}
-\alpha \sin ^{2} \theta+\beta \cos ^{2} \theta & -(\alpha+\beta) \sin \theta \cos \theta \\
0 & 0
\end{array}\right) .
$$

So $\sigma(\pi(A B))=\left\{-\alpha \sin ^{2} \theta+\beta \cos ^{2} \theta, 0\right\}$. Since $[0, \beta] \subseteq[-\alpha, \beta]=$ $\left\{-\alpha \sin ^{2} \theta+\beta \cos ^{2} \theta: \theta \in[0,2 \pi]\right\},[0, \beta] \subseteq \sigma(A B)$. Since $\sigma(A B)$ is rotation invariant, $\sigma(A B)$ contains the closed disc with center 0 and radius $\beta$. By Lemma 3.5, $\sigma(A B)$ is the closed disc of complex plane with center 0 and radius $\beta$.

Case 2. Both $A$ and $B$ are invertible. By Lemma 3.3 and Remark 3.4, we may assume that $A=\left(\begin{array}{cc}0 & 1 \\ \beta_{1} & 0\end{array}\right)$ and $B=\left(\begin{array}{cc}0 & 1 \\ \beta_{2} & 0\end{array}\right)$ such that $\beta_{1}, \beta_{2} \geq 1$. Then 
$A^{-1}=\left(\begin{array}{cc}0 & \beta_{1}^{-1} \\ 1 & 0\end{array}\right)$ and $B^{-1}=\left(\begin{array}{cc}0 & \beta_{2}^{-1} \\ 1 & 0\end{array}\right)$. We need to prove that $\sigma(A B)=$ $\left[1, \beta_{1} \beta_{2}\right] \times_{p}[0,2 \pi]$. By Lemma 3.5, $r(A B)=\beta_{1} \beta_{2}$ and $r\left((A B)^{-1}\right)=1$. This implies that $\sigma(A B) \subseteq\left[1, \beta_{1} \beta_{2}\right] \times_{p}[0,2 \pi]$. So we need only to prove $\sigma(A B) \supseteq\left[1, \beta_{1} \beta_{2}\right] \times_{p}[0,2 \pi]$.

For $\phi, \psi \in[0,2 \pi]$, let $U=\left(\begin{array}{cc}\cos \psi & e^{i \phi} \sin \psi \\ -\sin \psi & e^{i \phi} \cos \psi\end{array}\right)$. Then $U$ is a unitary matrix. Let $\pi_{1}(X)=U X U^{*}$ and $\pi_{2}(Y)=Y$ be $*$-representations of $M_{2}(\mathrm{C}) * 1$ and $1 * M_{2}(\mathrm{C})$ to $M_{2}(\mathrm{C})$, respectively. Let $\pi=\pi_{1} * \pi_{2}$ be the induced $*-$ representation of $\AA$ to $M_{2}(\mathrm{C})$. Then

$$
\pi(A B)=\left(\begin{array}{cc}
-\beta_{1} \beta_{2} e^{i \phi} \sin ^{2} \psi+\beta_{2} e^{-i \phi} \cos ^{2} \psi & * \\
* & \beta_{1} e^{i \phi} \cos ^{2} \psi-e^{-i \phi} \sin ^{2} \psi
\end{array}\right) .
$$

Let $\lambda_{1}(\phi, \psi), \lambda_{2}(\phi, \psi)$ be the eigenvalues of $\pi(A B)$. Then

$$
\begin{aligned}
& \lambda_{1}(\phi, \psi) \lambda_{2}(\phi, \psi)=\operatorname{det}(\pi(A B))=\operatorname{det}(A) \operatorname{det}(B)=\beta_{1} \beta_{2}, \\
& \begin{array}{l}
\lambda_{1}(\phi, \psi)+\lambda_{2}(\phi, \psi) \\
\quad=\left(\beta_{1} e^{i \phi}+\beta_{2} e^{-i \phi}\right) \cos ^{2} \psi-\left(\beta_{1} \beta_{2} e^{i \phi}+e^{-i \phi}\right) \sin ^{2} \psi
\end{array}
\end{aligned}
$$

Note that $\sigma(A B) \supseteq\left\{\lambda_{1}(\phi, \psi): \phi, \psi \in[0,2 \pi]\right\}$. We only need to prove that $\left\{\lambda_{1}(\phi, \psi): \phi, \psi \in[0,2 \pi]\right\} \supseteq\left[1, \beta_{1} \beta_{2}\right] \times_{p}[0,2 \pi]$. For this purpose, we need to show for every $r \in\left[1, \beta_{1} \beta_{2}\right], \theta \in[0,2 \pi]$, there are $\phi, \psi \in[0,2 \pi]$ such that

$$
r e^{i \theta}+\frac{\beta_{1} \beta_{2}}{r} e^{-i \theta}=\left(\beta_{1} e^{i \phi}+\beta_{2} e^{-i \phi}\right) \cos ^{2} \psi-\left(\beta_{1} \beta_{2} e^{i \phi}+e^{-i \phi}\right) \sin ^{2} \psi
$$

Let $\alpha=\cos ^{2} \psi$. Simple computations show that equation (3.3) is equivalent to the following

$$
\begin{array}{r}
\left(r+\frac{\beta_{1} \beta_{2}}{r}\right) \cos \theta+i\left(r-\frac{\beta_{1} \beta_{2}}{r}\right) \sin \theta=\left(\alpha\left(1+\beta_{1}\right)\left(1+\beta_{2}\right)-\left(1+\beta_{1} \beta_{2}\right)\right) \cos \phi \\
+i\left(\alpha\left(\beta_{1}-1\right)\left(\beta_{2}+1\right)+\left(1-\beta_{1} \beta_{2}\right)\right) \sin \phi
\end{array}
$$

Let

$$
\begin{aligned}
\Omega_{1}= & \left\{\left(r+\frac{\beta_{1} \beta_{2}}{r}\right) \cos \theta+i\left(r-\frac{\beta_{1} \beta_{2}}{r}\right) \sin \theta: r \in\left[1, \beta_{1} \beta_{2}\right], \theta \in[0,2 \pi]\right\}, \\
\Omega_{2}= & \left\{\left(\alpha\left(1+\beta_{1}\right)\left(1+\beta_{2}\right)-\left(1+\beta_{1} \beta_{2}\right)\right) \cos \phi\right. \\
& \left.+i\left(\alpha\left(\beta_{1}-1\right)\left(\beta_{2}+1\right)+\left(1-\beta_{1} \beta_{2}\right)\right) \sin \phi: \alpha \in[0,1], \phi \in[0,2 \pi]\right\} .
\end{aligned}
$$

Now we need only to prove $\Omega_{1}=\Omega_{2}$. Note that $\Omega_{1}$ is the union of a family of ellipses with center the origin point and semimajor axis and semiminor axis 
$\left|r+\frac{\beta_{1} \beta_{2}}{r}\right|$ and $\left|r-\frac{\beta_{1} \beta_{2}}{r}\right|, 1 \leq r \leq \beta_{1} \beta_{2}$, respectively. Similarly, $\Omega_{2}$ is the union of a family of ellipses with center the origin point and semimajor axis and semiminor axis $\left|\alpha\left(1+\beta_{1}\right)\left(1+\beta_{2}\right)-\left(1+\beta_{1} \beta_{2}\right)\right|$ and $\mid \alpha\left(\beta_{1}-1\right)\left(\beta_{2}+1\right)+(1-$ $\left.\beta_{1} \beta_{2}\right) \mid, 0 \leq \alpha \leq 1$, respectively. Note that the "largest" ellipse in $\Omega_{1}$ is with semimajor axis and semiminor axis $\left|1+\beta_{1} \beta_{2}\right|$ and $\left|\beta_{1} \beta_{2}-1\right|$, respectively; the "smallest" ellipse in $\Omega_{1}$ is with semimajor axis and semiminor axis $2 \sqrt{\left|\beta_{1} \beta_{2}\right|}$ and 0 , respectively. The "largest" ellipse in $\Omega_{2}$ is with semimajor axis and semiminor axis $\left|1+\beta_{1} \beta_{2}\right|$ and $\left|\beta_{1} \beta_{2}-1\right|$, respectively; the "smallest" ellipse in $\Omega_{2}$ is with semimajor axis and semiminor axis 0 and $\frac{2 \beta_{1}\left(\beta_{2}-1\right)}{\beta_{1}+1}$. So both $\Omega_{1}$ and $\Omega_{2}$ are the closure of the domain enclosed by the ellipse with center the origin point and semimajor axis and semiminor axis $\left|1+\beta_{1} \beta_{2}\right|$ and $\left|\beta_{1} \beta_{2}-1\right|$, respectively. Thus $\Omega_{1}=\Omega_{2}$.

\section{R-diagonal operators in $\mathscr{M}$}

In this section, we prove the following result. We will use the notation introduced in section 2.4.

Theorem 4.1. In $\mathcal{M}$, let $A \in M_{2}(\mathrm{C})_{(1)}$ and $B \in M_{2}(\mathrm{C})_{(2)}$. Then $A B$ is an $\mathrm{R}$-diagonal operator if and only if $\tau(A)=\tau(B)=0$.

To prove Theorem 4.1, we need the following lemmas.

Lemma 4.2. $\left\{W_{1}, V_{1}, W_{3} V_{3}\right\}^{\prime \prime} \cong L\left(\mathrm{Z}_{2}\right) * L\left(\mathrm{Z}_{2}\right) * L(\mathrm{Z})$.

Proof. Let $U=W_{3} V_{3}$. Then $U$ is a Haar unitary operator. We need only to prove that $U$ is * free with the von Neumann subalgebra generated by $W_{1}$ and $V_{1}$. Let $g_{1} g_{2} \cdots g_{n}$ be an alternating product of $\left\{U^{n}: n \neq 0\right\}$ and $\left\{W_{1}, V_{1}, W_{1} V_{1}, V_{1} W_{1}, W_{1} V_{1} W_{1}, V_{1} W_{1} V_{1}, \ldots\right\}$. By regrouping, it is an alternating product of $\left\{W_{1}, W_{1} W_{3}, W_{3}^{*} W_{1}, W_{3}^{*} W_{1} W_{3}, W_{3}, W_{3}^{*}\right\}$ and $\left\{V_{1}, V_{3} V_{1}\right.$, $\left.V_{1} V_{3}^{*}, V_{3} V_{1} V_{3}^{*}, V_{3}, V_{3}^{*}\right\}$. Thus the trace is 0 .

Lemma 4.3. $\left(\begin{array}{cc}0 & \alpha_{1} \\ \beta_{1} & 0\end{array}\right)_{(1)}\left(\begin{array}{cc}0 & \alpha_{2} \\ \beta_{2} & 0\end{array}\right)_{(2)}$ is an R-diagonal operator.

Proof. Note that

$$
\begin{aligned}
\left(\begin{array}{cc}
0 & \alpha_{1} \\
\beta_{1} & 0
\end{array}\right)_{(1)}\left(\begin{array}{cc}
0 & \alpha_{2} \\
\beta_{2} & 0
\end{array}\right)_{(2)} & \\
& =\left(\begin{array}{cc}
\alpha_{1} & 0 \\
0 & \beta_{1}
\end{array}\right)_{(1)}\left(\begin{array}{ll}
0 & 1 \\
1 & 0
\end{array}\right)_{(1)}\left(\begin{array}{ll}
0 & 1 \\
1 & 0
\end{array}\right)_{(2)}\left(\begin{array}{cc}
\beta_{2} & 0 \\
0 & \alpha_{2}
\end{array}\right)_{(2)} .
\end{aligned}
$$

By Lemma 4.2 and basic properties of R-diagonal operators given in 2.2, we prove the lemma. 
LemMA 4.4. With the assumption of Theorem 4.1 and assume $A B$ is an R-diagonal operator and $\tau\left(A^{2}\right) \neq 0$. Then $\tau(B)=0$.

Proof. Since $A B$ is an R-diagonal operator, $\tau(A B)=0$. Since $A, B$ are *-free, $\tau(A) \tau(B)=\tau(A B)=0$. If $\tau(B)=0$, then done. Otherwise, assume $\tau(A)=0$. Then $0=\tau(A B A B)=\tau\left(A^{2} B\right) \tau(B)=\tau\left(A^{2}\right)(\tau(B))^{2}$. By the assumption, $\tau(B)=0$.

LeMmA 4.5. Let $B \in M_{2}(\mathrm{C})_{(2)}$ and $\lambda$ be a complex number. Then $\sigma\left(E_{12} B\right)=\sigma\left(E_{12}(\lambda+B)\right)$.

Proof. By Jacobson's theorem,

$$
\begin{aligned}
\sigma\left(E_{12}(\lambda+B)\right) \cup\{0\} & =\sigma\left(E_{11} E_{12}(\lambda+B)\right) \cup\{0\} \\
& =\sigma\left(E_{12}(\lambda+B) E_{11}\right) \cup\{0\} \\
& =\sigma\left(E_{12} B E_{11}\right) \cup\{0\}=\sigma\left(B E_{12}\right) \cup\{0\} .
\end{aligned}
$$

Proof of Theorem 4.1. If $\tau(A)=\tau(B)=0$, then by Lemma 3.3 and Lemma 4.3, $A B$ is an R-diagonal operator. Conversely, assume that $A B$ is an R-diagonal operator. Then $0=\tau(A B)=\tau(A) \cdot \tau(B)$. So either $\tau(A)=0$ or $\tau(B)=0$. Without loss of generality, we assume that $\tau(A)=0$. If $\tau\left(A^{2}\right) \neq 0$, then $\tau(B)=0$ by Lemma 4.4. If $\tau\left(A^{2}\right)=0$, then $A$ is unitary equivalent to $\alpha E_{12}$. We may assume that $A=E_{12}$. By Theorem 2.1, if $E_{12} B$ is an Rdiagonal operator, then $\left(r\left(E_{12} B\right)\right)^{2}=\tau\left(B^{*} E_{21} E_{12} B\right)=\tau\left(E_{21} E_{12} B B^{*}\right)=$ $\left\|E_{12}\right\|_{2}^{2} \cdot\|B\|_{2}^{2}$. Note that $E_{12}(B-\tau(B))$ is an R-diagonal operator, $\left(r\left(E_{12}(B-\right.\right.$ $\tau(B)))^{2}=\left\|E_{12}\right\|_{2}^{2} \cdot\|B-\tau(B)\|_{2}^{2}$. By Lemma 4.5, $\|B\|_{2}^{2}=\|B-\tau(B)\|_{2}^{2}$. This implies that $\tau(B)=0$. This ends the proof.

Combining Theorem 4.1, Theorem 2.1 and the S-transform of Voiculescu (see [16], [17]), we have the following theorem (It is interesting to compare the following theorem and Theorem 3.6).

Theorem 4.6. Let $A \in M_{2}(\mathrm{C})_{(1)}, B \in M_{2}(\mathrm{C})_{(2)}$ and $\tau(A)=\tau(B)=0$. Then

(1) $\mu_{A B}$ is rotation invariant;

(2) $\sigma(A B)=\operatorname{supp} \mu_{A B}=\left[\left\|A^{-1}\right\|_{2}^{-1}\left\|B^{-1}\right\|_{2}^{-1},\|A\|_{2}\|B\|_{2}\right] \times_{p}[0,2 \pi]$;

(3) $\mu_{A B}(\{0\})=\max \left\{\mu_{A^{*} A}(\{0\}), \mu_{B^{*} B}(\{0\})\right\}$ and

$$
\mu_{A B}\left(\mathrm{~B}\left(0,\left(S_{\mu_{A^{*} A}} S_{\mu_{B^{*} B}}(t-1)\right)^{-1 / 2}\right)\right)=t, \quad \text { for } t \in\left[\mu_{A B}(\{0\}), 1\right] .
$$




\section{Algebraic techniques}

For $X \in \mathscr{M}$, define

$$
\Phi(X)=\left(\begin{array}{ll}
E_{11} X E_{11} & E_{11} X E_{21} \\
E_{12} X E_{11} & E_{12} X E_{21}
\end{array}\right) .
$$

Then $\Phi$ is a $*$-isomorphism from $\mathscr{M}$ onto $E_{11} \mathscr{M} E_{11} \otimes M_{2}(\mathrm{C})_{(1)}$. We will identify $\mathscr{M}$ with $E_{11} \mathscr{M} E_{11} \otimes M_{2}(\mathrm{C})_{(1)}$ by the canonical isomorphism $\Phi$. In [4], $\mathrm{K}$. Dykema proved that $E_{11} \mathscr{M} E_{11} \cong L\left(\mathrm{~F}_{3}\right)$. For $B \in M_{2}(\mathrm{C})_{(2)}$, we may write

$$
B=\left(\begin{array}{ll}
b_{11} & b_{12} \\
b_{21} & b_{22}
\end{array}\right)
$$

with respect to matrix units in $\mathscr{M}_{1}$. Then $b_{i j} \in L\left(\mathrm{~F}_{3}\right)$. In this section, we will develop the algebraic techniques used in [4]. Combining the matrix techniques, we may explicitly express $b_{i j}$ in terms of free generators of $L\left(\mathrm{~F}_{3}\right)$.

Let $\Lambda\left\{W_{1}, V_{1}\right\}$ be the set of words generated by $W_{1}, V_{1}$. Note that $W_{1}^{2}=$ $V_{1}^{2}=1$ and $\tau\left(W_{1}\right)=\tau\left(V_{1}\right)=0$. The following observation is crucial in [4]. The proof is an easy exercise.

Lemma 5.1. $\tau\left(g_{1} g_{2} \cdots g_{n}\right)=0$ for an alternating product of $\Lambda\left\{W_{1}, V_{1}\right\} \backslash$ $\left\{1, W_{1}\right\}$ and $\left\{E_{12}, E_{21}\right\}$.

Recall that $P=E_{11}$ and $Q=F_{11}$. Let $W$ be the "polar" part of $(1-P) Q P$ and $U=E_{12} W$. The following corollary is a special case of Theorem 3.5 of [4].

Corollary 5.2. $U$ is a Haar unitary operator in $\mathcal{M}_{P}=P \mathscr{M} P$ and $U$, $P Q P$ are $*$-free in $\mathcal{M}_{P}$.

With the canonical identification of $\mathscr{M}$ with $\mathscr{M}_{P} \otimes M_{2}(\mathrm{C})_{(1)}$,

$$
Q=\left(\begin{array}{cc}
P Q P & \sqrt{P Q P-(P Q P)^{2}} U \\
U^{*} \sqrt{P Q P-(P Q P)^{2}} & U^{*}(1-P Q P) U
\end{array}\right) .
$$

By [16], the distribution of $P Q P$ (relative to $\mathcal{M}_{P}$ ) is non-atomic and the density function is

$$
\rho(t)=\frac{1}{\pi} \frac{1}{\sqrt{\frac{1}{4}-\left(\frac{1}{2}-t\right)^{2}}}, \quad 0 \leq t \leq 1 .
$$

By Corollary 5.2, the von Neumann subalgebra $\mathscr{M}_{1}$ generated by $M_{2}(\mathrm{C})_{(1)}$ and $Q$ is $*$-isomorphic to $L\left(\mathrm{~F}_{2}\right) \otimes M_{2}(\mathrm{C})_{(1)}$. Since $\mathscr{M}_{1}$ is also $*$-isomorphic to 
$M_{2}(\mathrm{C}) * L\left(\mathrm{Z}_{2}\right), M_{2}(\mathrm{C}) * L\left(\mathrm{Z}_{2}\right) \cong L\left(\mathrm{~F}_{2}\right) \otimes M_{2}(\mathrm{C})$, which is proved by Dykema in [4].

Since $V_{1}=2 Q-1$

$$
V_{1}=\left(\begin{array}{cc}
2 P Q P-1 & 2 \sqrt{P Q P-(P Q P)^{2}} U \\
2 U^{*} \sqrt{P Q P-(P Q P)^{2}} & U^{*}(1-2 P Q P) U
\end{array}\right) .
$$

Simple computation shows that the density function of $2 P Q P-1$ is

$$
\sigma(t)=\frac{1}{\pi} \frac{1}{\sqrt{1-t^{2}}}, \quad-1 \leq t \leq 1 .
$$

Let $H=2 P Q P-1$, then

$$
V_{1}=\left(\begin{array}{cc}
H & \sqrt{1-H^{2}} U \\
U^{*} \sqrt{1-H^{2}} & -U^{*} H U
\end{array}\right) .
$$

Let $H=V|H|$ be the polar decomposition of $H$. Since $H$ is a symmetric selfadjoint operator, $V^{2}=1$ and $V$ is independent with the von Neumann algebra generated by $|H|$ in the classical probability sense. Let $h=|H|, u=$ $V U, v=U V$. Then $u, v$ are Haar unitary operators and the distribution of $h$ relative to $\mathcal{M}_{P}$ is non-atomic.

LEMMA 5.3. $h, u, v$ are $*$ free.

Proof. Let $g_{1} g_{2} \cdots g_{n}$ be an alternating product of elements of $\mathbb{\subseteq}=$ $\{|H|\}^{\prime \prime} \ominus \mathrm{CI},\left\{(V U)^{n}: n \neq 0\right\},\left\{(U V)^{n}: n \neq 0\right\}$. By regrouping, it is an alternating product of elements of $\{\Im, V, V \Subset, \subseteq V, V \Subset V\}$ and $\left\{U^{n}: n \neq 0\right\}$. Since $H$ and $U$ are $*$-free, $\{\Im, V, V \Subset, \subseteq V, V \subseteq V\}$ and $\left\{U^{n}: n \neq 0\right\}$ are free. Since $V$ and $\Im$ are independent, $\tau(V S)=\tau(S V)=0$ for $S \in \widetilde{\Xi}$. This implies that $\tau\left(g_{1} g_{2} \cdots g_{n}\right)=0$.

By simple computations, we have the following:

$$
\begin{aligned}
V_{1} E_{11} V_{1} & =\left(\begin{array}{cc}
h^{2} & \sqrt{1-h^{2}} h u \\
u^{*} h \sqrt{1-h^{2}} & u^{*}\left(1-h^{2}\right) u
\end{array}\right) \\
V_{1} E_{12} V_{1} & =\left(\begin{array}{cc}
H U^{*} \sqrt{1-H^{2}} & -H U^{*} H U \\
U^{*} \sqrt{1-H^{2}} U^{*} \sqrt{1-H^{2}} & -U^{*} \sqrt{1-H^{2}} U^{*} H U
\end{array}\right) \\
& =\left(\begin{array}{cc}
h v^{*} \sqrt{1-h^{2}} & -h v^{*} h u \\
u^{*} \sqrt{1-h^{2}} v^{*} \sqrt{1-h^{2}} & -u^{*} \sqrt{1-h^{2}} v^{*} h u
\end{array}\right) .
\end{aligned}
$$


By Lemma 2.2, $\mathscr{M} \cong M_{2}(\mathrm{C})_{(1)} *\left(V_{1} M_{2}(\mathrm{C})_{(1)} V_{1}\right) \cong \mathcal{M}_{P} \otimes M_{2}(\mathrm{C})_{(1)}$. With this isomorphism, $\mathscr{M}_{P}$ is the von Neumann algebra generated by $h, u$ and $v$ by (5.2) and (5.4). So $\mathcal{M}_{P} \cong L\left(\mathrm{~F}_{3}\right)$. By simple computations, we have

$$
V_{1}\left(\begin{array}{ll}
\alpha & \beta \\
\gamma & \sigma
\end{array}\right)_{(1)} V_{1}=\left(\begin{array}{ll}
b_{11} & b_{12} \\
b_{21} & b_{22}
\end{array}\right)
$$

where

$$
\begin{aligned}
& b_{11}=\sigma+(\alpha-\sigma) h^{2}+\gamma \sqrt{1-h^{2}} v h+\beta h v^{*} \sqrt{1-h^{2}}, \\
& b_{12}=(\alpha-\sigma) h \sqrt{1-h^{2}} u+\gamma \sqrt{1-h^{2}} v \sqrt{1-h^{2}} u-\beta h v^{*} h u, \\
& b_{21}=(\alpha-\sigma) u^{*} h \sqrt{1-h^{2}}-\gamma u^{*} h v h+\beta u^{*} \sqrt{1-h^{2}} v^{*} \sqrt{1-h^{2}}, \\
& b_{22}=\alpha+(\sigma-\alpha) u^{*} h^{2} u-\gamma u^{*} h v \sqrt{1-h^{2}} u-\beta u^{*} \sqrt{1-h^{2}} v^{*} h u .
\end{aligned}
$$

THEOREM 5.4. $\mathscr{M} \cong L\left(\mathrm{~F}_{3}\right) \otimes M_{2}(\mathrm{C})_{(1)}$; furthermore, let $B=\left(\begin{array}{cc}\alpha & \beta \\ \gamma & \sigma\end{array}\right)_{(2)}$ in $M_{2}(\mathrm{C})_{(2)}$, then with respect to the matrix units $\left\{E_{i j}\right\}_{i, j=1,2} \subset M_{2}(\mathrm{C})_{(1)}$, $B=\left(\begin{array}{ll}b_{11} & b_{12} \\ b_{21} & b_{22}\end{array}\right)_{(1)}$, where $b_{i j}$ are given as above.

EXAmPLE 5.5. In Theorem 5.4, let $\beta=\gamma=0$. Then we have

$$
\left(\begin{array}{cc}
\alpha & 0 \\
0 & \sigma
\end{array}\right)_{(2)}=\left(\begin{array}{cc}
\sigma+(\alpha-\sigma) h^{2} & (\alpha-\sigma) h \sqrt{1-h^{2}} u \\
(\alpha-\sigma) u^{*} h \sqrt{1-h^{2}} & \alpha+(\sigma-\alpha) u^{*} h^{2} u
\end{array}\right)_{(1)} .
$$

ExAmPLE 5.6. In Theorem 5.4, let $\alpha=\sigma$ and $\gamma=0$. Then we have

$$
\left(\begin{array}{ll}
\alpha & \beta \\
0 & \alpha
\end{array}\right)_{(2)}=\left(\begin{array}{cc}
\alpha+\beta h v^{*} \sqrt{1-h^{2}} & -\beta h v^{*} h u \\
\beta u^{*} \sqrt{1-h^{2}} v^{*} \sqrt{1-h^{2}} & \alpha-\beta u^{*} \sqrt{1-h^{2}} v^{*} h u
\end{array}\right)_{(1)} .
$$

REMARK 5.7. By equation (5.2), the distribution of $h^{2}$ is the distribution of $E_{11} V_{1} E_{11} V_{1} E_{11}$ relative to $M_{P}$. So the distribution of $h^{2}$ is same as the distribution of $P Q P$ (relative to $\mathcal{M}_{P}$ ). By [16], the distribution of $P Q P$ (relative to $\mathscr{M}_{P}$ ) is non-atomic and the density function is

$$
\rho(t)=\frac{1}{\pi} \frac{1}{\sqrt{\frac{1}{4}-\left(\frac{1}{2}-t\right)^{2}}}, \quad 0 \leq t \leq 1 .
$$




\section{Miscellaneous examples}

ExAmple 6.1. We compute the Brown spectrum of $\alpha E_{12}+\beta F_{12}$. Let $F_{12}=$ $\left(\begin{array}{ll}0 & 1 \\ 0 & 0\end{array}\right)_{(2)}=\left(\begin{array}{ll}b_{1} & b_{2} \\ b_{3} & b_{4}\end{array}\right)_{(1)}$. Then

$$
\begin{aligned}
\left(\alpha E_{12}+\beta F_{12}\right)^{2}=\alpha \beta\left(E_{12} F_{12}+F_{12} E_{12}\right) & =\alpha \beta\left(E_{12}+F_{12}\right)^{2} \\
& =\alpha \beta\left(\begin{array}{cc}
b_{3} & b_{1}+b_{4} \\
0 & b_{3}
\end{array}\right)_{(1)} .
\end{aligned}
$$

So $\mu_{\left(\alpha E_{12}+\beta F_{12}\right)^{2}}=\mu_{\alpha \beta b_{3}}$. By equation (5.3), the distribution of $b_{3}$ is same as the distribution of $\left(U^{*} \sqrt{1-H^{2}}\right)^{2}$. Since $U^{*} \sqrt{1-H^{2}}$ is an R-diagonal operator, $\left(U^{*} \sqrt{1-H^{2}}\right)^{2}$ is also an R-diagonal operator. Since the distribution of $\alpha E_{12}+$ $\beta F_{12}$ is rotation invariant, $\mu_{\alpha E_{12}+\beta F_{12}}=\mu_{\sqrt{|\alpha \beta| b}}$, where $b=U^{*} \sqrt{1-H^{2}}$. Simple computations show that (or by Proposition 5.10 and Corollary 5.11 of [5])

$$
d \mu_{b}(z)=\frac{1}{\pi} \frac{1}{\left(1-r^{2}\right)^{2}} d r d \theta, \quad 0 \leq r \leq \frac{1}{\sqrt{2}} .
$$

Hence

$$
d \mu_{\alpha E_{12}+\beta F_{12}}(z)=d \mu_{\sqrt{|\alpha \beta| b}}(z)=\frac{1}{\pi} \frac{|\alpha \beta|^{3 / 2}}{(|\alpha \beta|-r)^{2}} d r d \theta, \quad 0 \leq r \leq \sqrt{\frac{|\alpha \beta|}{2}}
$$

and

$$
\sigma\left(\alpha E_{12}+\beta F_{12}\right)=\overline{\mathrm{B}\left(0, \sqrt{\frac{|\alpha \beta|}{2}}\right)} .
$$

Corollary 6.2. $r\left(\alpha E_{12}+\beta F_{12}\right)=\sqrt{\frac{|\alpha \beta|}{2}}$.

Corollary 6.3. Let $A \in M_{2}(\mathrm{C})_{(1)}$ and $B \in M_{2}(\mathrm{C})_{(2)}$. Then $A+B$ is an $\mathrm{R}$-diagonal operator if and only if $A+B=0$.

Proof. Indeed, if $A+B$ is an R-diagonal operator, then $\tau(A+B)=0$. So we may assume that $\tau(A)=\tau(B)=0$. Let $\lambda,-\lambda$ and $\eta,-\eta$ be the spectra of $A$ and $B$, respectively. Then $0=\tau\left((A+B)^{2}\right)=\tau\left(A^{2}\right)+\tau\left(B^{2}\right)=\lambda^{2}+\eta^{2}$. By simple computation we have $0=\tau\left((A+B)^{4}\right)=\tau\left(A^{4}\right)+\tau\left(B^{4}\right)+$ $4 \tau\left(A^{2}\right) \tau\left(B^{2}\right)=\lambda^{4}+\eta^{4}+4 \lambda^{2} \eta^{2}=\left(\lambda^{2}+\eta^{2}\right)^{2}+2 \lambda^{2} \eta^{2}=2 \lambda^{2} \eta^{2}$. Thus $\lambda=\eta=0$. This implies that $A$ and $B$ are unitary equivalent to $\alpha E_{12}$ and $\beta F_{12}$, respectively. By Corollary 6.2,r $(A+B)=\sqrt{\frac{|\alpha \beta|}{2}}$. On the other hand, since we assume that $A+B$ is an R-diagonal operator, by Theorem 2.1, $(r(A+B))^{2}=$ 
$\tau\left(\left(A^{*}+B^{*}\right)(A+B)\right)=\tau\left(\left(\bar{\alpha} E_{21}+\bar{\beta} F_{21}\right)\left(\alpha E_{12}+\beta F_{12}\right)\right)=\frac{|\alpha|^{2}+|\beta|^{2}}{2}$. So $|\alpha|^{2}+|\beta|^{2}=|\alpha \beta|$. This implies that $\alpha=0$ and $\beta=0$. Hence $A+\stackrel{2}{B}=0$.

EXAMPLE 6.4. We compute the spectrum and Brown spectrum of

$$
X=\left(\begin{array}{ll}
1 & 0 \\
0 & 0
\end{array}\right)_{(1)}\left(\begin{array}{ll}
\alpha & \beta \\
0 & \alpha
\end{array}\right)_{(2)} .
$$

By Example 5.6, we have the following

$$
\begin{aligned}
X & =\left(\begin{array}{ll}
1 & 0 \\
0 & 0
\end{array}\right)_{(1)}\left(\begin{array}{cc}
\alpha & \beta \\
0 & \alpha
\end{array}\right)_{(2)} \\
& =\left(\begin{array}{ll}
1 & 0 \\
0 & 0
\end{array}\right)_{(1)}\left(\begin{array}{cc}
\alpha+\beta h v^{*} \sqrt{1-h^{2}} & -\beta h v^{*} h u \\
\beta u^{*} \sqrt{1-h^{2}} v \sqrt{1-h^{2}} & \alpha-\beta u^{*} \sqrt{1-h^{2}} v^{*} h u
\end{array}\right)_{(1)} \\
& =\left(\begin{array}{cc}
\alpha+\beta h v^{*} \sqrt{1-h^{2}} & -\beta h v^{*} h u \\
0 & 0
\end{array}\right)_{(1)}
\end{aligned}
$$

So $\sigma(X)=\{0\} \cup \sigma\left(\alpha+\beta h v^{*} \sqrt{1-h^{2}}\right)$ and $\mu_{X}=\frac{1}{2} \delta_{0}+\frac{1}{2} \mu_{\alpha+\beta h v^{*} \sqrt{1-h^{2}}}$. Note that $\mu_{h v^{*} \sqrt{1-h^{2}}}=\mu_{v^{*} \sqrt{1-h^{2}} h}$ and $v^{*} \sqrt{1-h^{2}} h$ is an R-diagonal operator. We have the following computations:

$$
\begin{aligned}
\left\|\sqrt{1-h^{2}} h\right\|_{2}^{2} & =\tau_{P}\left(\left(1-h^{2}\right) h^{2}\right)=\tau_{P}((1-P Q P) P Q P) \\
& =\int_{0}^{1} \frac{1}{\pi} \frac{t(1-t) d t}{\sqrt{\frac{1}{4}-\left(\frac{1}{2}-t\right)^{2}}}=\frac{1}{8} \\
\left\|\left(\sqrt{1-h^{2}} h\right)^{-1}\right\|_{2}^{2} & =\tau_{P}\left(\left(\left(1-h^{2}\right) h^{2}\right)^{-1}\right)=\tau_{P}\left(((1-P Q P) P Q P)^{-1}\right) \\
& =\int_{0}^{1} \frac{1}{\pi} \frac{d t}{t(1-t) \sqrt{\frac{1}{4}-\left(\frac{1}{2}-t\right)^{2}}}=\infty .
\end{aligned}
$$

By Theorem 2.1,

$$
\sigma(X)=\operatorname{supp} \mu_{X}=\{0\} \cup \overline{\mathrm{B}(\alpha,|\beta| / 2 \sqrt{2})} .
$$

EXAMPLE 6.5. We compute the spectrum and Brown spectrum of

$$
Y=\left(\begin{array}{ll}
0 & 1 \\
0 & 0
\end{array}\right)_{(1)}\left(\begin{array}{cc}
\alpha & 0 \\
0 & \beta
\end{array}\right)_{(2)} .
$$


By Example 5.5, we have the following

$$
\begin{aligned}
Y & =\left(\begin{array}{ll}
0 & 1 \\
0 & 0
\end{array}\right)_{(1)}\left(\begin{array}{cc}
\alpha & 0 \\
0 & \beta
\end{array}\right)_{(2)} \\
& =\left(\begin{array}{ll}
0 & 1 \\
0 & 0
\end{array}\right)_{(1)}\left(\begin{array}{cc}
\beta+(\alpha-\beta) h^{2} & (\alpha-\beta) h \sqrt{1-h^{2}} u \\
(\alpha-\beta) u^{*} h \sqrt{1-h^{2}} & \alpha+(\beta-\alpha) u^{*} h^{2} u
\end{array}\right)_{(1)} \\
& =\left(\begin{array}{cc}
(\alpha-\beta) u^{*} h \sqrt{1-h^{2}} & \alpha+(\beta-\alpha) u^{*} h^{2} u \\
0 & 0
\end{array}\right)_{(1)}
\end{aligned}
$$

Since $u^{*} h \sqrt{1-h^{2}}$ is an R-diagonal operator, similar computations as Example 6.4, we have

$$
\sigma(Y)=\operatorname{supp} \mu_{Y}=\overline{\mathrm{B}(0,|\alpha-\beta| / 2 \sqrt{2})} .
$$

EXAMPLE 6.6. We compute the spectrum and Brown spectrum of

$$
Z=\left(1+\alpha E_{12}\right)\left(1+\beta F_{12}\right)=\left(\begin{array}{cc}
1 & \alpha \\
0 & 1
\end{array}\right)_{(1)}\left(\begin{array}{ll}
1 & \beta \\
0 & 1
\end{array}\right)_{(2)}
$$

For $\lambda \in \mathrm{C}$, we have

$$
\begin{aligned}
Z-\lambda 1 & =\left(1+\alpha E_{12}\right)\left(1+\beta F_{12}\right)-\lambda\left(1+\alpha E_{12}\right)\left(1-\alpha E_{12}\right) \\
& =\left(1+\alpha E_{12}\right)\left(\lambda \alpha E_{12}+\beta F_{12}-(\lambda-1)\right) .
\end{aligned}
$$

This implies that $\lambda \in \sigma(Z)$ if and only if $\lambda-1 \in \sigma\left(\lambda \alpha E_{12}+\beta F_{12}\right)$. By Example 6.1, $\lambda-1 \in \sigma\left(\lambda \alpha E_{12}+\beta F_{12}\right)$ if and only if

$$
|\lambda-1|^{2} \leq \frac{|\alpha \beta||\lambda|}{2}
$$

So

$$
\sigma(Z)=\left\{\lambda \in \mathrm{C}:|\lambda-1|^{2} \leq \frac{|\alpha \beta||\lambda|}{2}\right\}
$$

In the following, we will show that supp $\mu_{Z} \supseteq \partial \sigma(Z)=\left\{\lambda \in C:|\lambda-1|^{2}=\right.$ $\left.\frac{|\alpha \beta||\lambda|}{2}\right\}$. For this purpose, we need only to prove that supp $\mu_{Z-1} \supseteq \partial \sigma(Z-1)=$ $\left\{\lambda \in \mathrm{C}:|\lambda|^{2}=\frac{|\alpha \beta||\lambda+1|}{2}\right\}$. 
Note that $\Delta\left(1+\alpha E_{12}\right)=1$. For $\lambda \in \mathrm{C}$, we have

$$
\begin{aligned}
\log \Delta & ((Z-1)-\lambda) \\
& =\log \Delta\left(\left(1+\alpha E_{12}\right)\left(1+\beta F_{12}\right)-(1+\lambda)\left(1+\alpha E_{12}\right)\left(1-\alpha E_{12}\right)\right) \\
& =\log \Delta\left(1+\alpha E_{12}\right)+\log \Delta\left(1+\beta F_{12}-\lambda+(1+\lambda) \alpha E_{12}\right) \\
& =\log \Delta\left((1+\lambda) \alpha E_{12}+\beta F_{12}-\lambda\right) .
\end{aligned}
$$

By Example 6.1, $\mu_{(1+\lambda) \alpha E_{12}+\beta F_{12}}=\mu_{\sqrt{|1+\lambda||\alpha \beta| b}}$. Hence,

$$
\begin{aligned}
\log \Delta & ((Z-1)-\lambda) \\
& =\log \Delta(\sqrt{|1+\lambda||\alpha \beta|} b-\lambda) \\
& =\log \Delta\left(b-\frac{\lambda}{\sqrt{|1+\lambda||\alpha \beta|}}\right)-\log \frac{|\lambda|}{\sqrt{|1+\lambda||\alpha \beta|}}+\log |\lambda| .
\end{aligned}
$$

Since $b$ is an R-diagonal operator, this implies that

(6.1) $\log \Delta\left(b-\frac{|\lambda|}{\sqrt{|1+\lambda||\alpha \beta|}}\right)$

$$
=\log \frac{|\lambda|}{\sqrt{|1+\lambda||\alpha \beta|}}-\log |\lambda|+\log \Delta((Z-1)-\lambda) .
$$

Suppose $\lambda_{0} \in \partial \sigma(Z-1)$ and $\lambda_{0} \notin \operatorname{supp} \mu_{Z-1}$. Then there is $\delta>0$ such that $\mathrm{B}\left(\lambda_{0}, \delta\right) \subset \mathrm{C} \backslash \operatorname{supp} \mu_{Z-1}$. Now $\log \Delta((Z-1)-\lambda)$ is a harmonic function on $\mathrm{B}\left(\lambda_{0}, \delta\right)$. Since $\tau\left((Z-1)^{n}\right)=0$ for all $n=1,2, \ldots$ By Lemma 4.3 of [8], for $\lambda \in C$ such that $|\lambda| \geq r(Z-1), \log \Delta((Z-1)-\lambda)=\log |\lambda|$. By the uniqueness of harmonic functions, we have $\log \Delta((Z-1)-\lambda)=\log |\lambda|$ for $\lambda \in \mathrm{B}\left(\lambda_{0}, \delta\right)$. By equation (6.1), this implies that

$$
\log \Delta\left(b-\frac{|\lambda|}{\sqrt{|1+\lambda||\alpha \beta|}}\right)=\log \frac{|\lambda|}{\sqrt{|1+\lambda||\alpha \beta|}} .
$$

Let $r=\frac{|\lambda|}{\sqrt{|1+\lambda||\alpha \beta|}}$. Then equation (6.2) implies that

$$
\log \Delta(b-r)=\log r
$$

for $r \in(s, t) \subseteq\left[0, \frac{1}{\sqrt{2}}\right]$. Since $b$ is an R-diagonal operator, this implies that $\log \Delta(b-z)$ is harmonic on the annulus with inner radius $s$ and outer radius $t$, $0<s<t<\frac{1}{\sqrt{2}}$. By Theorem 2.1, supp $\mu_{b}=\overline{\mathrm{B}\left(0, \frac{1}{\sqrt{2}}\right)}$. It is a contradiction. 


\section{Hyperinvariant subspaces for operators in $\mathscr{M}$}

Lemma 7.1. For $X \in \mathcal{M}$, if $\operatorname{supp} \mu_{X}=\{\lambda\}$, then $\tau\left(X^{n}\right)=\lambda^{n}$ for $n=1,2, \ldots$.

Proof. $\tau\left(X^{n}\right)=\int_{\operatorname{supp} \mu_{X}} z^{n} d \mu_{X}(z)=\lambda^{n}$.

The converse of Lemma 7.1 is not true. Since for an R-diagonal operator $X$, we have $\tau\left(X^{n}\right)=0$ for $n=1,2, \ldots$

Proposition 7.2. Let $X=A+B$, where $A \in M_{2}(\mathrm{C})_{(1)}$ and $B \in M_{2}(\mathrm{C})_{(2)}$. If $A, B$ are not scalar matrices, then supp $\mu_{X}$ contains more than two points.

Proof. Suppose $A, B$ are not scalar matrices. Since $A+B=\tau(A) 1+$ $\tau(B) 1+(A-\tau(A) 1)+(B-\tau(B) 1)$, to show supp $\mu_{X}$ contains more than two points, we need only to show that $\mu_{(A-\tau(A) 1)+(B-\tau(B) 1)}$ contains more than two points. So we may assume that $\tau(A)=\tau(B)=0$ and $A, B \neq 0$. Assume that the spectra of $A$ and $B$ are $\lambda_{1},-\lambda_{1}$ and $\lambda_{2},-\lambda_{2}$. If $\tau\left(A^{2}\right)=\tau\left(B^{2}\right)=0$, then $A$ and $B$ are unitarily equivalent to $\alpha E_{12}$ and $\beta F_{12}$ in $M_{2}(\mathrm{C})_{(1)}$ and $M_{2}(\mathrm{C})_{(2)}$, respectively. Thus $\mu_{X}=\mu_{\alpha E_{12}+\beta F_{12}}$. By Example 6.1, supp $\mu_{X}$ contains more than two points. Now suppose $\tau\left(A^{2}\right) \neq 0$ or $\tau\left(B^{2}\right) \neq 0$. Without loss of generality, we assume that $\lambda_{1}^{2}=\tau\left(A^{2}\right) \neq 0$. Note that $\tau(A+B)=0$ and $\tau\left((A+B)^{2}\right)=\tau\left(A^{2}\right)+\tau\left(B^{2}\right)=\lambda_{1}^{2}+\lambda_{2}^{2}$. If $\lambda_{1}^{2}+\lambda_{2}^{2} \neq 0$, by Lemma 7.1, supp $\mu_{X}$ contains more than two points. Suppose $\lambda_{1}^{2}+\lambda_{2}^{2}=0$. Then $\tau\left(B^{2}\right)=$ $\lambda_{2}^{2} \neq 0$. Simple computations show that

$$
\begin{aligned}
\tau\left((A+B)^{4}\right) & =\tau\left(A^{4}\right)+\tau\left(B^{4}\right)+4 \tau\left(A^{2}\right) \tau\left(B^{2}\right) \\
& =\lambda_{1}^{4}+\lambda_{2}^{4}+4 \lambda_{1}^{2} \lambda_{2}^{2}=\left(\lambda_{1}^{2}+\lambda_{2}^{2}\right)^{2}+2 \lambda_{1}^{2} \lambda_{2}^{2}=2 \lambda_{1}^{2} \lambda_{2}^{2} \neq 0 .
\end{aligned}
$$

Note that $\tau(A+B)=0$. By Lemma 7.1, supp $\mu_{X}$ contains more than two points.

Proposition 7.3. Let $X=A B$, where $A \in M_{2}(\mathrm{C})_{(1)}$ and $B \in M_{2}(\mathrm{C})_{(2)}$. If $A, B$ are not scalar matrices, then supp $\mu_{X}$ contains more than two points.

Proof. Suppose $A, B$ are not scalar matrices. We consider the following cases:

Case 1. $\tau(A)=\tau(B)=0$ and $A, B \neq 0$. By Theorem 4.1, $A B(\neq 0)$ is an R-diagonal operator. So supp $\mu_{X}$ contains more than two points.

Case 2. $\tau(A)=0, \tau(B) \neq 0$ or $\tau(A) \neq 0, \tau(B)=0$. Without loss of generality, we assume that $\tau(A)=0$ and $\tau(B) \neq 0$. Then $\tau(A B)=0$ and $\tau(A B A B)=\tau\left(A^{2}\right) \tau(B)$. If $\tau\left(A^{2}\right) \neq 0$, then $\tau(A B A B) \neq 0$. By Lemma 7.1, supp $\mu_{X}$ contains more than two points. If $\tau\left(A^{2}\right)=0$, then $A$ is unitarily equivalent to $\alpha E_{12}$ in $M_{2}(C)_{(1)}$. By Lemma $4.5, \mu_{X}=\mu_{\alpha E_{12}(B-\tau(B))}$. Since 
$\alpha E_{12}\left(B-\tau(B)(\neq 0)\right.$ is an R-diagonal operator, supp $\mu_{X}$ contains more than two points.

Case 3. $\tau(A) \neq 0$ and $\tau(B) \neq 0$. We may assume that $\tau(A)=\tau(B)=1$. Let $A=1+A_{1}$ and $B=1+B_{1}$. Then $\tau\left(A_{1}\right)=\tau\left(B_{1}\right)=0$.

Subcase 3.1. $\tau\left(A_{1}^{2}\right) \neq 0$ or $\tau\left(B_{1}^{2}\right) \neq 0$. We may assume that $\tau\left(A_{1}^{2}\right) \neq 0$. Simple computation shows that $\tau(A B)=1, \tau(A B A B)=1+\tau\left(A_{1}^{2}\right)+\tau\left(B_{1}^{2}\right)$ and $\tau\left((A B)^{3}\right)=1+3\left(\tau\left(A_{1}^{2}\right)+\tau\left(B_{1}^{2}\right)\right)+9 \tau\left(A_{1}^{2}\right) \tau\left(B_{1}^{2}\right)$. If $\tau\left(A_{1}^{2}\right)+\tau\left(A_{2}^{2}\right) \neq 0$, then $\tau(A B A B) \neq 1$. By Lemma 7.1, supp $\mu_{X}$ contains more than two points. If $\tau\left(A_{1}^{2}\right)+\tau\left(A_{2}^{2}\right)=0$, then $\tau\left(A_{2}^{2}\right)=-\tau\left(A_{1}^{2}\right) \neq 0$. So $\tau\left((A B)^{3}\right) \neq 1$. By Lemma 7.1 again, supp $\mu_{X}$ contains more than two points.

Subcase 3.2. $\tau\left(A_{1}^{2}\right)=\tau\left(A_{2}^{2}\right)=0$. Then $A_{1}$ and $A_{2}$ are unitarily equivalent to $\alpha E_{12}$ and $\beta F_{12}$ in $M_{2}(\mathrm{C})_{(1)}$ and $M_{2}(\mathrm{C})_{(2)}$, respectively. So $\mu_{X}=$ $\mu_{\left(1+\alpha E_{12}\right)\left(1+\beta F_{12}\right)}$. We may assume that $A=\left(\begin{array}{cc}1 & \alpha \\ 0 & 1\end{array}\right)_{(1)}$ and $B=\left(\begin{array}{ll}1 & \beta \\ 0 & 1\end{array}\right)_{(2)}$. By Example 6.6, supp $\mu_{X}$ contains more than two points.

Corollary 7.4. Let $X=A B$ or $X=A+B$, where $A \in M_{2}(\mathrm{C})_{(1)}$ and $B \in M_{2}(C)_{(2)}$. If $X \neq \lambda 1$, then $X$ has a nontrivial hyperinvariant subspace relative to $\mathcal{M}$.

Proof. If $X=A+B$ and $A=\lambda 1$ or $B=\lambda 1$, then $X=\lambda 1+B$ or $X=\lambda 1+A$. If $X$ is not a scalar matrix and $\eta$ is an eigenvalue of $X$, then $\operatorname{ker}(X-\eta 1)$ is a nontrivial hyperinvariant subspace of $X$. If $X=A+B$ and $A, B \neq \lambda 1$, then supp $\mu_{X}$ contains more than two points by Proposition 7.3. By [9], $X$ has a nontrivial hyperinvariant subspace relative to $\mathscr{M}$. If $X=A B$ and $A=\lambda 1$ or $B=\lambda 1$, then $X=\lambda B$ or $X=\lambda A$. If $X$ is not a scalar matrix and $\eta$ is an eigenvalue of $X$, then $\operatorname{ker}(X-\eta 1)$ is a nontrivial hyperinvariant subspace of $X$. If $X=A B$ and $A, B \neq \lambda 1$, then supp $\mu_{X}$ contains more than two points. By [9], $X$ has a nontrivial hyperinvariant subspace relative to $\mathscr{M}$.

ACKNOWLEDGEMENTs. The authors want to express their deep gratitude to professor Eric Nordgren for valuable discussions. The authors also thank the referee for some useful suggestions.

\section{REFERENCES}

1. Biane, P., Lehner, F., Computation of some examples of Brown's spectral measure in free probability, Colloq. Math. 90 (2001), 181-211.

2. Brown, L. G., Lidskii's theorem in the type II case, Geometric methods in operator algebras, H. Araki and E. Effros (Eds.) Pitman Res. Notes Math. Ser 123 (1986), 1-35.

3. Ching, W. M., Free products of von Neumann algebras, Trans. Amer. Math. Soc. 178 (1973), $147-163$.

4. Dykema, K., Interpolated free group factors, Pacific J. Math. 163 (1994), 123-135. 
5. Fang, J., Hadwin, D., Mohan, R., On transitive algebras containing a standard finite von Neumann subalgebra, J. Funct. Anal. 252 (2007), 581-602.

6. Fuglede, B., and Kadsion, R. V., Determinant theory in finite factors, Ann. of Math. 55 (3)(1952), 520-530.

7. Greenleaf, F. P., Invariant Means on Topological Groups and their Applications, Van Nostrand Mathitatical Studies 16, Van Nostrand Reinhold Co., New York-Toronto, Ont.-London, 1969

8. Haagerup, U., and Larsen, F., Brown's spectral distribution measure for R-diagonal elements in finite von Neumann algebras, J. Funct. Anal. 176 (2000), 331-367.

9. Haagerup, U., and Schultz, H., Invariant Subspaces for Operators in a General $\mathrm{II}_{1}$-factor, preprint available at http://www.arxiv.org/pdf/math.OA/0611256.

10. Haagerup, U., and Schultz, H., Brown measures of unbounded operators affiliated with a finite von Neumann algebra, Math. Scand. 100 (2007), 209-263.

11. Larsen, F., Powers of R-diagonal elements, J. Operator Theory 47 (2002), no. 1, 197-212.

12. Nica, A., and Speicher, R., R-diagonal pairs - a common approach to Haar unitaries and circular elements, Fields Inst. Commun. (1997), 149-188.

13. Nica, A., and Speicher, R., Commutators of free random variables, Duke. Math. J. 92(3) (1998), 553-592.

14. Radjavi, H., and Rosenthal, P., Invariant Subspaces, Springer-Verlag, New York, 1973.

15. Schultz, H., Brown measures of sets of commuting operators in a $\mathrm{II}_{1}$-factor, J. Funct. Anal. 236 (2006), 457-489.

16. Voiculescu, D. V., Multiplication of certain noncommuting random variables, J. Operator Theory 18 (1987), no. 2, 223-235.

17. Voiculescu, D. V., Dykema, K., and Nica, A., Free Random Variables, CRM Monogr. Ser. 1 (1992).

DEPARTMENT OF MATHEMATICS

UNIVERSITY OF NEW HAMPSHIRE

DURHAM, NH 03824

USA

E-mail: jfang@cisunix.unh.edu,don@math.unh.edu
HEBEI UNIVERSITY OF TECHNOLOGY GUANGRONGDAO, HONGQIAO DISTRICT TIANJIN 300130

CHINA

E-mail: mxjsusan@hebut.edu.cn 